\title{
EFFECT OF INTRAVENOUS DEXMEDETOMIDINE PREMEDICATION ON CHARACTERISTICS OF BUPIVACAINE SPINAL BLOCK
}

\author{
Anita Mathew ${ }^{1}$, Ivan Koshy², Mary Verghese3, Narjeet Kaur4 \\ ${ }_{1}^{1}$ Assistant Professor, Department of Anaesthesiology, Believer's Church Medical College and Hospital, Thiruvalla, Kerala, India. \\ ${ }^{2}$ Assistant Professor, Department of Anaesthesiology, Believer's Church Medical College and Hospital, Thiruvalla, Kerala, India. \\ 3 Professor and HOD, Department of Anaesthesiology, Believer's Church Medical College and Hospital, Thiruvalla, Kerala, India. \\ ${ }^{4}$ Professor, Department of Anaesthesiology, Christian Medical College, Ludhiana, Punjab, India.
}

\section{BACKGROUND}

ABSTRACT

Regional anaesthesia has gained worldwide popularity and in the complexity of modern anaesthesiology it offers many advantages: simplicity, rapid onset, dense motor block and avoidance of the potential airway complications related to general anaesthesia. It produces analgesia with minimal physiological disturbance.

Aim- This is a study to evaluate the effect of intravenous dexmedetomidine premedication on subarachnoid block using $0.5 \%$ bupivacaine.

\section{MATERIALS AND METHODS}

This prospective study was conducted in a tertiary care hospital in Northern India over a one-year period among patients undergoing infraumbilical surgeries. This was a randomised, double-blinded placebo-controlled study on 60 patients belonging to American Society of Anesthesiologists (ASA) physical status I or II, aged 18 - 55 years. Patients received either dexmedetomidine $0.5 \mu / \mathrm{kg}$ (Group D) or saline intravenously (Group C) five minutes before spinal anaesthesia with $0.5 \%$ bupivacaine. The maximum level of sensory block, duration of block, sensory and motor regression time and sedation were recorded. The time for first analgesic requirement in the post-operative period was also noted. Side effects, if any, were also noted.

\section{RESULTS}

Patients who received IV dexmedetomidine had faster onset and delayed recovery of motor blockade. In addition, the level of sensory block achieved was higher and the time for sensory regression was longer in dexmedetomidine arm. However, the grade of motor blockade was comparable in both groups. The dexmedetomidine arm also had higher sedation and better post-operative analgesia with longer time interval for rescue analgesia. Side effects were comparable in both the groups.

\section{CONCLUSION}

We conclude that IV dexmedetomidine is a suitable premedicant drug in patients receiving subarachnoid block.

\section{KEY WORDS}

Spinal Anaesthesia, Dexmedetomidine, Bupivacaine.

HOW TO CITE THIS ARTICLE: Mathew A, Koshy I, Verghese M, et al. Effect of intravenous dexmedetomidine premedication on characteristics of bupivacaine spinal block. J. Evolution Med. Dent. Sci. 2018;7(43):4633-4638, DOI: 10.14260/jemds/2018/1034

\section{BACKGROUND}

Regional anaesthesia has gained worldwide popularity and in the complexity of modern anaesthesiology it offers many advantages- simplicity, rapid onset, dense motor block and avoidance of the potential airway complications related to general anaesthesia. It produces analgesia with minimal physiological disturbance.

Various drugs have been tried through oral, intravenous and intrathecal route to prolong the duration of block, analgesia and sedation in patients undergoing various surgeries under spinal anaesthesia.

Alpha-2 adrenergic mechanism of analgesia has been exploited for more than 100 years. Dexmedetomidine is a

'Financial or Other Competing Interest': None.

Submission 14-09-2018, Peer Review 08-10-2018,

Acceptance 15-10-2018, Published 22-10-2018.

Corresponding Author:

Dr. Ivan Koshy,

Department of Anaesthesiology.

Believer's Church Medical College and Hospital,

Thiruvalla, Kerala, India.

E-mail: drivankoshy@gmail.com

DOI: $10.14260 /$ jemds $/ 2018 / 1034$

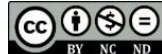

highly selective alpha-2 adrenoceptor agonist with both sedative and analgesic properties and is devoid of respiratory depressant effect. ${ }^{1}$ Dexmedetomidine produces analgesia by action at several sites; supraspinally at the level of the locus coeruleus (LC), where it decreases activation of neurons within the LC. ${ }^{2}$ This study was conducted to evaluate the role of $\alpha_{2}$-adrenoceptor agonist dexmedetomidine in anaesthetic practice as an intravenous premedicant in patients undergoing regional anaesthesia using bupivacaine.

We compared haemodynamics, sensory blockade, motor blockade, time for rescue analgesia and side effects of drugs or technique.

\section{MATERIALS AND METHODS}

After approval by the Institutional Ethics Committee, this study was conducted in the Department of Anaesthesia, at tertiary level hospital in northern India. Sixty patients of either sex belonging to American Society of Anesthesiologists (ASA), grade I or II, aged 18 - 55 years were included in this prospective, placebo controlled, randomised, double-blinded study. The sample size was taken as per convenience. Patients scheduled for elective infraumbilical surgeries, of less than 3 hours duration were enrolled. All patients were kept NPO $\geq 6$ hours and given Tab Diazepam $5 \mathrm{mg}$ at bedtime. 
Patients were randomly divided into two groups of 30 each. Patients were randomised into study and control groups using a set of random numbers from the random number table with allocation ratio of 1: 1 into either of the groups that is Group D and Group C. After the allocation had been made, the drug was drawn according to the serial order by an index anaesthesiologist (not a part of the study) who prepared either of the drugs. Both the patient and the observer recording study parameters were blinded to the drug being used. At the end of the study, decoding was done. The Group D patients received $0.5 \mu \mathrm{g} / \mathrm{kg}$ of dexmedetomidine diluted to a total volume of $5 \mathrm{~mL}$ and Group $\mathrm{C}$ received $5 \mathrm{~mL}$ of normal saline. Both were injected over 10 minutes as a single dose. Five minutes after the end of infusion patient was placed in lateral position at L2-L3/ L3-L4 position, subarachnoid block was given using $0.5 \%$ bupivacaine with $25-\mathrm{G}$ Quincke needle.

Pulse rate, blood pressure and oxygen saturation were recorded every 2 minutes till first ten minutes, then every 10 minutes till 1 hour followed by recording every 15 minutes till the end of surgery. Any decrease in heart rate below 50 beats per minute was noted and treated with incremental doses of injection Atropine $0.3 \mathrm{mg}$ intravenously. Episodes of hypotension (fall in systolic blood pressure $>20 \%$ of baseline value) was treated with incremental doses of ephedrine $6 \mathrm{mg}$ intravenously. Sensory blockade was assessed using pinprick test in the midaxillary line. Motor block was assessed using Modified Bromage Score 3 (Appendix 1). Sensory and motor block was assessed every two minutes for the first ten minutes and thereafter every ten minutes during surgery and post-operatively. The highest sensory block level and its onset time were noted. Recovery time for sensory and motor block was to be recorded. Recovery time for sensory blockade was noted as two dermatome regression of anaesthesia from the maximum level.

Patients were assessed for pain score by performing Visual Analogue Self Rating Method. The scale consisted of a $10 \mathrm{~cm}$ horizontal line connecting points marked as "no pain at all" and worst possible pain.

Patients were assessed for pain every 30 minutes after surgery till the patient complained of moderate pain. Patients with a Visual Analogue Score (VAS) of 3 or more received Inj. Diclofenac $75 \mathrm{mg}$ intramuscularly for rescue analgesia. The time for the first request for rescue analgesia and the number of patients who required supplemental analgesia was recorded. Duration of effective analgesia was measured as the time from onset of analgesia to the patient's first request for supplemental analgesic administration. The Ramsay sedation score was used for grading sedation. The score was reevaluated every 10 minutes for upto 120 minutes. Side effects such as nausea, vomiting, hypotension and bradycardia were noted.

\section{Statistical Analysis}

The statistical analysis was carried out using Statistical Package for Social Sciences (SPSS Inc., Chicago, IL, version 16.0 for Windows). All quantitative variables were estimated using measures of central location (Mean, Median) and measures of dispersion (Standard deviation). For normally distributed data means were compared using student's t-test for two groups. Qualitative or categorical variables were described as frequencies and proportions. Proportions were compared using Chi-square or Fisher's exact test, whichever was applicable. All statistical tests were two-sided and performed at a significance level of $\alpha=.05$.

\section{RESULTS}

Patients of both groups were statistically comparable regarding mean age, weight, gender, ASA physical status and surgical characteristics.

There was statistically significant fall from baseline in pulse rate readings in both the groups. On intergroup comparison, the fall in the pulse rate was significantly more in Group D from 8 to 60 mins ( $p=0.004)$ (Graph 1). The maximum blood pressure fall was observed at 10 minutes in both the groups. On intergroup comparison, the fall in the systolic blood pressure was significantly more in Group D $(\mathrm{p}=0.000)$ (Graph 2). Maximum sensory level was higher in Group D as compared to Group C ( $p=.002)$ (Graph 3). Time for sensory regression of two dermatomes was significantly extended in patients of Group D (2.88 $\pm 0.77 \mathrm{hrs}$.) as compared to Group C (2.47 \pm 0.358 hrs.) ( $\mathrm{p}=000)$ (Graph 4).

Onset of motor block, there was statistical difference between both groups $(\mathrm{p}=0.001)$. On intergroup comparison, Group D had faster onset of motor blockade as compared to Group C (Graph 5).

In Group I the mean motor recovery time was $3.260 \pm$ 0.39 , whereas in Group C the mean recovery time was 2.817 hours and SD of 0.3075 (Maximum recovery time was 3.5 hours and minimum 2 hours) ( $\mathrm{p}=0.000)$ (Graph 6).

The time taken for recovery was more in Group D compared to Group C. In Group D, the mean time for rescue analgesia was 5 hours with Standard Deviation (SD) of 1.03 (Maximum time was 7 hours and minimum was 4 hours), whereas in Group C the mean time was 4.13 hours and SD of 0.95 (maximum time was 6 hours and minimum 3 hours) The time taken for rescue analgesia was more in Group D as compared to C (Graph 7).

On intergroup comparison sedation score was significantly higher in Group D at 10, 20, 30, 40, 50, 60, 70 and 80 minutes ( $p$ value $<0.05$ ) (Graph 8 ).

Nausea/ vomiting- None of the patients in both groups had nausea and vomiting.

Hypotension- In Group D 14 (46.2\%) had hypotension, whereas in Group C 11 (36.6\%) patients had hypotension.

Bradycardia- In Group D 2 (6.6\%) patients had bradycardia, whereas in Group C $3(10 \%)$ patients had bradycardia. Any other complications- In Group D 2 (6.6\%) patients had arrhythmia, whereas in Group C 1 (3.3\%) patient had arrhythmia and 2 (6.6\%) patients had shivering (Graph9).

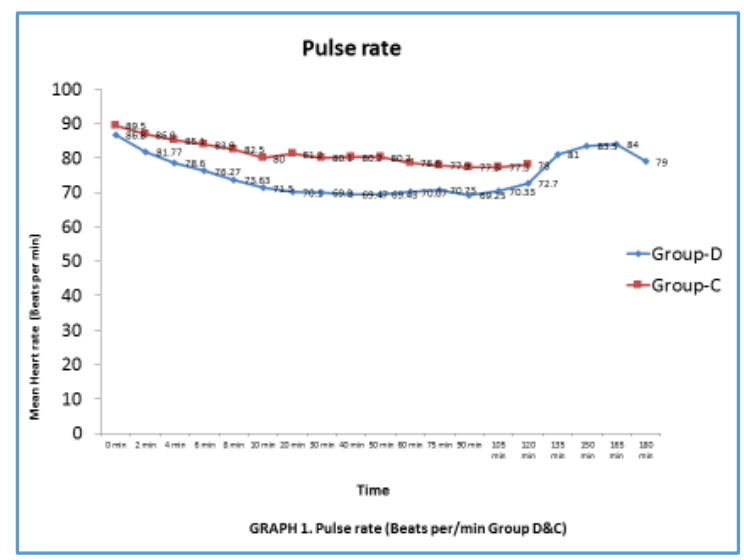



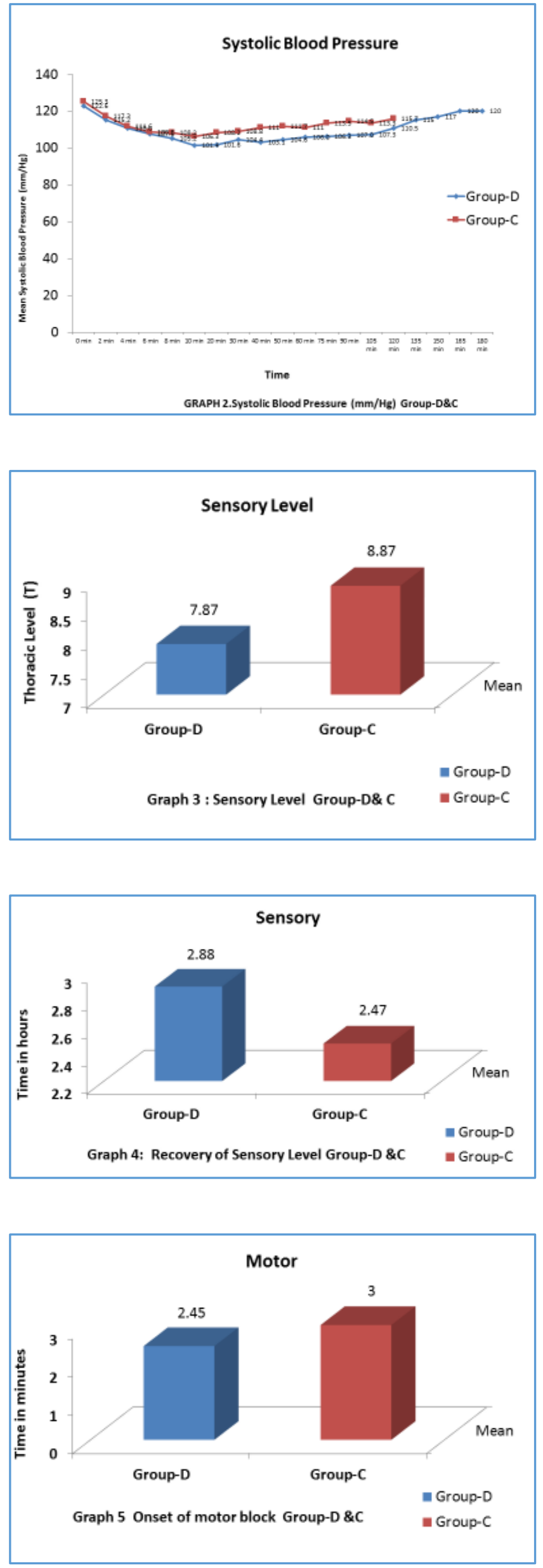
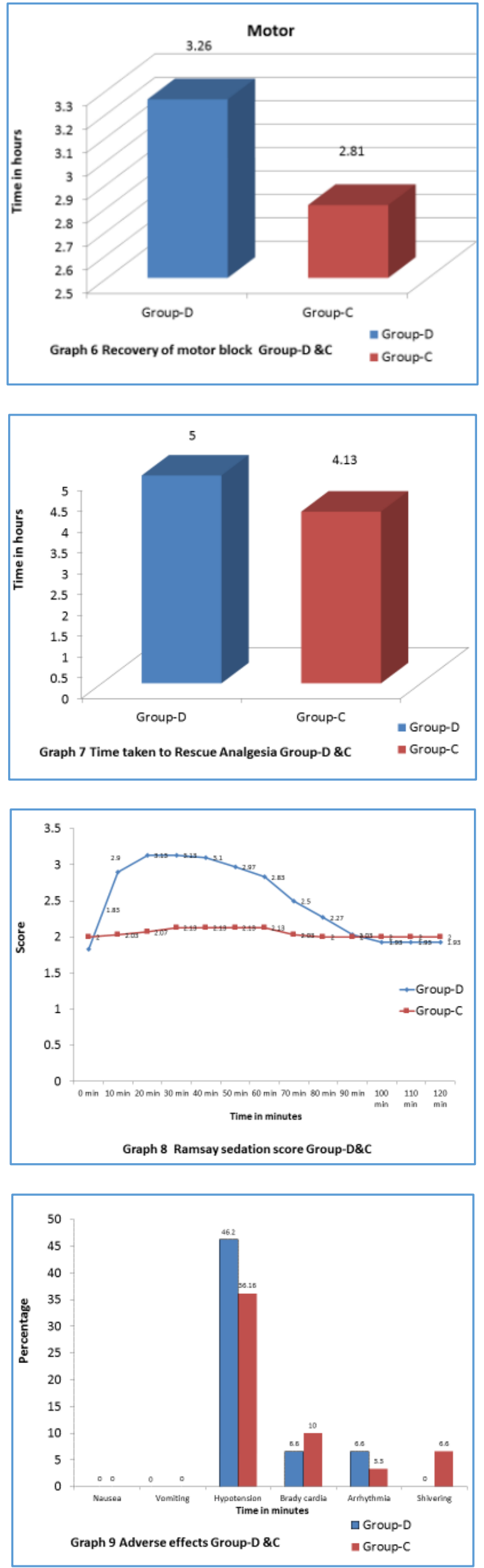


\section{DISCUSSION}

In our study in Group D the mean age of patients was 32.9 years with Standard Deviation (SD) of 11.45, whereas in Group C mean age was 36.0 years and SD of 12.20 (p-value= 0.280). In Group D mean weight was $65.00 \mathrm{~kg}$ with Standard Deviation (SD) of 9.39, whereas in Group C the mean weight was $64.70 \mathrm{~kg}$ and SD of 17.63 respectively ( $p$ value $=0.892$ ). The age and weight of the patients in both the groups were comparable and there was no statistically significant difference between Group D and C in the demographic parameters. The duration of surgery was also comparable in both the groups.

Mahmoud M Al-Mustafa et $\mathrm{al}^{3}$ in their prospective study of 48 cases compared to intravenous dexmedetomidine and normal saline following spinal isobaric bupivacaine. In their study the mean age in dexmedetomidine group was 64.2 years +/- 9.7 and in normal saline group it was 64.7 years +/12.5 (p value- 0.87 ). The mean BMI was $28.1 \pm 4.6$ in the normal saline group and in dexmedetomidine group 26.6 \pm 3.7 ( $p$ value- 0.23 ). The age, weight and duration of surgery of the both the groups were comparable in their study, which correlates with our study.

In our study the haemodynamic parameters studied included heart rate and blood pressure. In Group D, the mean baseline heart rate was 86.8 per minute with standard deviation of 12.4. The pulse rate showed continuous fall and maximum fall was observed at 90 minutes. Thereafter, it marginally increased till 120 minutes, but it remained significantly below baseline $(p<0.001)$. In this group, the number of patients in whom surgery lasted beyond 120 minutes was insignificant for use of statistical purposes.

In Group C, the mean baseline pulse rate was 89.53 per minute with a standard deviation of 15.067. In this group also, there was continuous fall in the pulse rate. The maximum fall was observed at 105 minutes. In this group, for none of the patients, surgery lasted beyond 120 minutes. On intergroup comparison, the fall in the pulse rate was significantly more in Group I at 8, 10, 20, 30, 40, 50, 60 mins $(\mathrm{p}=0.004)$.

In Group D, the baseline systolic blood pressure was $122.67 \mathrm{mmHg}$ with standard deviation of 14.606 . The fall in systolic blood pressure from the baseline value was statistically significant from 2 minutes to 120 minutes $(\mathrm{p}<0.001-0.004)$.

In Group C, the mean baseline systolic blood pressure was $125.33 \mathrm{mmHg}$ with Standard deviation of 11.366 . There was a fall in systolic blood pressure at 2, 4, 6, 8, 10, 20, 30, 40, 50, $60,75,90,105$ and 120 minutes which was statistically significant $(\mathrm{p}<0.000-0.045)$ with means ranging from 117.33 to 115.71 . The maximum fall was observed at 10 minutes in both the groups. On intergroup comparison, the fall in the systolic blood pressure was significantly more in Group D at 8, 10, 20, 30, 40, 50, 60, 75 and 90 minutes $(\mathrm{p}=0.000)$.

Mahmoud $\mathrm{M}$ et $\mathrm{al}^{3}$ in their study used dexmedetomidine 1 $\mu \mathrm{g} / \mathrm{kg}$ loading dose followed by $0.5 \mu \mathrm{g} / \mathrm{kg} / \mathrm{hr}$ following spinal anaesthesia. They also observed that the heart rate was significantly decreased in dexmedetomidine group as compared to saline group in the first hour. Thus, our findings correlates with their study. But they did not observe any significant difference in the mean arterial pressure. Coskuner et $\mathrm{al}^{4}$ used intravenous dexmedetomidine infusion for sedation in bupivacaine epidural block and compared it with physiological saline infusion. They reported that heart rate was significantly lower in dexmedetomidine group than saline group. .

In Group D, the mean level of sensory block was thoracic (T)- 7.87 with Standard Deviation (SD) of 1.252, whereas in Group C the mean level was T- 8.87 and SD of 1.106 $(\mathrm{p}=0.002)$.

The mean recovery time was 2.88 hours with Standard Deviation (SD) of 0.77 in Group D. In Group C, the mean recovery time was 2.47 hours and SD of 0.358 .

There was statistically significant difference between the two groups in both level of sensory block and recovery time. Level achieved was higher and the time for sensory regression was longer in Group D as compared to Group C. Kaya et $\mathrm{al}^{5}$ also observed that maximum upper level of sensory block was higher with dexmedetomidine (T $4.6 \pm 0.6$ ) than with midazolam group (T $6.4 \pm 0.9$ ) or with saline group ( $\mathrm{T} 6.4 \pm 0.8$ ). Time for sensory regression was longer in dexmedetomidine group than in midazolam or the saline groups. Coskuner et $\mathrm{al}^{4}$ also observed significant longer recovery time from sensory block in dexmedetomidine group. This correlates with our findings.

In Group D the mean onset time for motor blockade was 2.45 minutes with Standard Deviation (SD) of 0.575, whereas in Group C the mean onset was 3.00 minutes and SD of 0.683 $(p=0.001)$. On statistical analysis, Group D had faster onset of motor blockade as compared to Group C. Though the onset time for motor blockade was shorter in Group D, but the grade was comparable. In Group D, the mean recovery time from motor blockade was 3.260 hours with Standard deviation (SD) of 0.39 , whereas in Group C the mean recovery time was 2.817 hours and SD of 0.3075 .

On statistical analysis, the time taken for recovery from motor blockade was longer in Group D compared to Group C $(\mathrm{p}=0.000)$.

Mahmoud et $\mathrm{al}^{3}$ in their study observed that the time for regression to modified Bromage scale 0 was significantly prolonged in dexmedetomidine group in comparison with saline group. This correlates with our findings.

Kanazi et al ${ }^{6}$ compared the effect of $30 \mu \mathrm{gm}$ of intrathecal clonidine and $3 \mu \mathrm{gm}$ of dexmedetomidine as adjunct to spinal bupivacaine. They reported that in patients receiving dexmedetomidine the time to reach Bromage grade 3 motor block was significantly shorter than the control group who had received only intrathecal bupivacaine. The regression time to Bromage zero was significantly longer in dexmedetomidine group even when it is used intrathecally.

In our study, Ramsay sedation score was used for assessment of sedation. The score was re-evaluated every 10 minutes for upto 120 minutes. In Group D, sedation score increased from $1.83 \pm 0.379$ at zero minute to maximum of $3.13 \pm 0.346$ at 20 and 30 minutes. The maximum sedation score was observed from 20 to 40 minutes. In Group D sedation score was 2 at zero minute, remained the same throughout the observed period. The sedation score was significantly higher in Group D from 10 minutes to 80 minutes ( $p$ value $<0.05$ ). This infers that dexmedetomidine provides good sedation without respiratory depression, as none of the patients had any dip in the oxygen saturation. Kaya et $\mathrm{al}^{5}$ in their study used similar score for sedation. They found that the median range of the highest Ramsay sedation 
score was $2(2-5)$ in the dexmedetomidine group, 3 in midazolam group and 1 in saline group. Sedation score was greater in dexmedetomidine and midazolam group than saline group.

In both Group D and C, VAS score was zero upto 120 minutes. But from 120 to 180 minutes, VAS score was lower in Group D than in Group C indicating better post-operative analgesia in dexmedetomidine group.

Kaya et $\mathrm{al}^{5}$ observed that the overall 24-hour VAS scores were similar for the dexmedetomidine, midazolam and saline group. The VAS pain scores did not change over time (postoperative $4,8,12,24$ hours) in the three groups and were similar among groups.

In Group D the mean time for rescue analgesia was 5 hours with Standard Deviation (SD) of 1.03, whereas in Group C the mean time was 4.13 hours and SD of 0.95 . The time taken to rescue analgesia was more in Group D as compared to $\mathrm{C}$, which infers that dexmedetomidine reduces the requirement of analgesics in the post-operative period.

Kaya et $\mathrm{al}^{5}$ in their study observed that time to request for post-operative analgesia was longer in dexmedetomidine group than in the midazolam and saline groups ( $p$ value $<.001$ ) between the dexmedetomidine group and both the midazolam and saline group. This correlates with our findings.

In our study, we assessed for adverse effects too. None of the patients in both groups had nausea and vomiting. Mahmoud et $\mathrm{al}^{3}$ in their study reported that one patient had nausea and vomiting in the saline group, but none in dexmedetomidine group. The difference was not statistically significant.

In our study in Group D 46.2\% had hypotension, whereas in Group C 36.6\% patients had hypotension and both the groups were comparable ( $p$ value $=0.441$ ) .

Kaya et $\mathrm{al}^{5}$ in their study observed hypotension in $8 \%$ patients in dexmedetomidine group, whereas $16 \%$ patients in saline group and none in midazolam group. The difference was not statistically significant between both groups. Mahmoud et $\mathrm{al}^{3}$ found $16 \%$ patients had hypotension in saline group and none in dexmedetomidine. Difference was not statistically significant between both groups.

In our study in Group D 6.6\% patients had bradycardia, whereas in Group C 10\% patients had bradycardia. The incidence of bradycardia was also comparable (p-value= 0.647). Kaya et $\mathrm{al}^{5}$ in their study observed bradycardia in $8 \%$ patients, whereas 1 patient in saline group and none in midazolam group. Mahmoud et $\mathrm{al}^{3}$ in their study $8.3 \%$ patients had bradycardia in saline group and $16 \%$ patients had bradycardia. There was no statistically significant difference in both groups. This correlates with our study. In Group C, 2 (6.6\%) patients had shivering. Coskuner et al ${ }^{4}$ in their study found decreased incidence of shivering in dexmedetomidine group as compared to saline group.

In Group D 2 (6.6\%) patients had arrhythmia, whereas in Group C 1 (3.3\%) patient had arrhythmia.

Our results indicate that intravenous dexmedetomidine premedication prolonged the duration of bupivacaine induced sensory blockade during spinal anaesthesia and increased the maximum upper level of sensory block. In addition, it increased the time until first request for analgesic for post-operative pain relief and decreased the requirement of analgesic in the postoperative period. It also provided sedation without compromising respiration. Synergistic interaction between dexmedetomidine and local anaesthetics have been observed in previous studies. Memis et $\mathrm{al}^{7}$ reported that addition of $0.5 \mu \mathrm{g} / \mathrm{kg}$ dexmedetomidine to lignocaine for IVRA shortened the onset time and prolonged the sensory and motor block recovery time without causing side effect. Coskuner et $\mathrm{al}^{4}$ have shown that intravenous dexmedetomidine prolongs the recovery time of sensory blockade of bupivacaine epidural anaesthesia. Though Mahmoud et $\mathrm{al}^{3}$ and Kaya et $\mathrm{al}^{5}$ have reported that intravenous dexmedetomidine prolonged the duration of sensory block of bupivacaine spinal anaesthesia and the maximum upper level of sensory block. The supraspinal, direct analgesic and vasoconstricting actions of dexmedetomidine are suggested to be involved in this mechanism. We also observed adverse effects like hypotension, bradycardia, arrhythmia though present could be easily managed. Nevertheless, the relatively small number of patients included in our study warrants larger studies to conclusively evaluate the effect of intravenous dexmedetomidine premedication in bupivacaine spinal anaesthesia.

\section{CONCLUSION}

Intravenous dexmedetomidine significantly prolonged the duration of sensory and motor block of bupivacaine spinal anaesthesia. It provided good sedation without respiratory depression. Time for rescue analgesia was significantly prolonged with dexmedetomidine group and prevented shivering. However, dexmedetomidine caused decrease in heart rate and blood pressure. Limitation to this study was small sample size, its side effects and cost.

\section{ACKNOWLEDGEMENT}

I thank Dr. Valsa Abraham, the Head of Department of Anaesthesia, Christian Medical College, Ludhiana for allowing me to conduct my study. A special thanks to my beloved teachers and anaesthesia technicians.

\section{REFERENCES}

[1] Hall JE, Uhrich TD, Barney JA, et al. Sedative, amnesic, and analgesic properties of small-dose dexmedetomidine infusions. Anaesth Analg 2000;90(3):699-705.

[2] Gertler R, Brown HC, Mitchell DH, et al. Dexmedetomidine: a novel sedative-analgesic agent. Proc (Bayl Univ Med Cent) 2001;14(1):13-21.

[3] Al-Mustafa, Badran IZ, Abu-Ali HM, et al. Intravenous dexmedetomidine prolongs bupivacaine spinal analgesia. Middle East Journal of Anaesthesiology 2009;20(2):225-31.

[4] Coskuner I, Tekin M, Kati I, et al. Effects of dexmedetomidine on the duration of anaesthesia and wakefulness in bupivacaine epidural block. Eur J Anaesthesiol 2007;24(6):535-40.

[5] Kaya FN, Yavascaoglu B, Turker G, et al. Intravenous dexmedetomidine, but not midazolam, prolongs bupivacaine spinal anaesthesia. Can J Anaesth 2010;57(1):39-45. 
[6] Kanazi GE, Aouad MT, Jabbour-Khoury SI, et al. Effect of low-dose dexmedetomidine or clonidine on the characteristics of bupivacaine spinal block. Acta Anaesthesiol Scand 2006;50(2):222-7.
[7] Memis D, Turan A, Karamanlioglu B, et al. Adding dexmedetomidine to lidocaine for intravenous regional anaesthesia. Anaesth Analg 2004;98(3):83540. 